\title{
Multi-period integrated natural gas and electric power system probabilistic optimal power flow incorporating power-to-gas units
}

\author{
Guoqiang SUN ${ }^{1}$, Shuang CHEN ${ }^{1}$, Zhinong WEI ${ }^{1}$, Sheng CHEN ${ }^{1}$ \\ MPCE
}

\begin{abstract}
The increasing adoption of gas-fired power plants directly strengthens the coupling between electric power and natural gas systems. Current industrial practice in optimal power flow for electric power systems has not taken the security constraints of gas systems into consideration, resulting in an overly-optimistic solution. Meanwhile, the operation of electric power and natural gas systems is coupled over multiple periods because of the ramp rate limits of power generators and the slow dynamical characteristics of gas systems. Based on these motivations, we propose a multi-period integrated natural gas and electric power system probabilistic optimal power flow (M-GEPOPF) model, which includes dynamic gas flow models. To address the uncertainties originating from wind power and load forecasting, a probabilistic optimal power flow (POPF) calculation based on a three-point estimate method (3PEM) is adopted. Moreover, power-togas $(\mathrm{PtG})$ units are employed to avoid wind power curtailment and enable flexible bi-directional energy flows between the coupled energy systems. An integrated IEEE
\end{abstract}

CrossCheck date: 2 March 2017

Received: 2 October 2016/Accepted: 6 March 2017/Published online: 25 April 2017

(C) The Author(s) 2017. This article is an open access publication

$\triangle$ Shuang CHEN

hhuchenshuang@163.com

Guoqiang SUN

hhusunguoqiang@163.com

Zhinong WEI

wzn_nj@263.net

Sheng CHEN

chenshenghhu@163.com

1 College of Energy and Electrical Engineering, Hohai University, Nanjing 210098, China
RTS 24-bus electric power system and the Belgian 20-node natural gas system are employed as a test case to verify the applicability of the proposed M-GEPOPF model, and to demonstrate the potential economic benefits of PtG units.

Keywords Natural gas and electric power system, Network interdependency, Optimal power flow, Uncertainty, Power-to-gas unit

\section{Introduction}

With the longstanding large-scale exploitation and utilization of fossil fuels, the world is facing the formidable challenges of energy resource depletion and heavy pollution emissions. On 3 August 2015, the United States Environmental Protection Agency (EPA) announced the Clean Power Plan, representing a historic step in the reduction of carbon emissions from power plants [1]. Thus, adjusting the energy structure in the power sector has become an imperative aspect of the agenda for change. Natural gas has been playing an increasing role in bridging the gap between fossil fuels and renewable energies owing to three factors.

1) The retirement of coal units.

2) The economic efficiency and low-carbon emissions of natural gas units.

3) The rapid response of gas units for supporting volatile renewable energy sources. It is estimated that the natural gas market share of electricity will jump to $56 \%$, while coal drops by half to $21 \%$ in the U.S. by 2038 [2]. Consequently, the natural gas network is expected to be increasingly coupled with the electric power network. 
A current emphasis on this interdependency was provided by the FERC/NERC Staff Report on the 2011 Southwest Cold Weather Event [3] that included a discussion on the interdependency of the electric and natural gas industries. The report stated that utilities are becoming increasingly reliant on gas-fired generation, in large part because shale production has dramatically reduced the cost of gas. Likewise, compressors used in the gas industry are more likely than in the past to be powered with electricity, rather than gas. As a result, deficiencies in the supply of either electricity or natural gas affect not only consumers of that commodity, but of the other commodity as well. The report went on to state that any resolution of the many issues arising from electric and natural gas interdependency must be informed by an examination of more than one cold weather event in one part of the country and urged regulatory and industry bodies to explore solutions to the many interdependency problems which are likely to remain of concern in the future. As such, the security (or reliability) challenge resulting from the strong interdependency between electric power and gas systems requires a combined optimization and control regime for the coupled energy systems. In addition, reliable communication is critical to address this interdependency and electric-communications-natural gas networks are each dependent on the other to function properly.

Efforts to separately optimize the two networks can fail to capitalize on the potential economic benefits of an integrated energy network [4]. Moreover, far worse is that neglecting the interdependencies can lead to inappropriate decisions [5, 6]. A number of studies have focused on modeling integrated natural gas and electric power systems. An optimal power flow model for combined gas and electric networks, denoted as GEOPF, was proposed in [7-9]. Here, the primary elements in a gas network were modeled to resemble an electric system. However, electricity travels at the speed of light, while a natural gas system features considerably slower transient processes caused by load swings, leading to gas flow coupling in time and space. Consequently, efforts to optimize integrated gas and electric systems have increasingly employed multiperiod models. A detailed gas network model was formulated, and considerations of gas storage and line pack were introduced in [10, 11]. Moreover, it was argued that, although a steady-state model is applicable for an electric system, it is not applicable for gas systems due to the low gas velocity. Therefore, dynamic approximations for a gas system are required to guarantee a reliable optimal solution to an integrated model [12]. From this viewpoint, the optimization of an integrated gas and electric system has been recently conducted in the field of unit commitment [13]. However, most researchers have formulated integrated systems as a linear programming problem using linearized electric power/gas flow models to guarantee a global optimum solution. Despite the computational efficiency, the obtained solution may not be the representative of a solution to the original nonlinear problem. To the best of the authors' knowledge, multi-period integrated gas and electric system optimal power flow (M-GEOPF) has not been formulated based on precise nonlinear electric power and gas flow models.

On the other hand, renewable energy sources (e.g., wind power) are expected to be a key element for the transition to sustainable low-carbon energy systems [14]. In fact, wind energy and natural gas are complementary, and offer a number of possibilities for coordinated use. For example, gas-fired plants enhance the flexibility of electric systems, which can facilitate the increasing deployment of renewable energy. Nonetheless, the uncertainty and variability of wind power pose substantial challenges for electric power system operation. As such, the integration of large-scale wind farms with existing electric power systems necessitates conducting optimization analysis under uncertainty. In the literature, some studies have addressed wind power uncertainty. A robust optimization model was proposed for analyzing the interdependencies between gas and electric infrastructures, which led to an uncertainty-immunized, but conservative solution $[15,16]$. The operating strategies for an integrated gas and electric network obtained by twostage and multi-stage stochastic programming have also been presented [17]. However, the performance of the obtained solution was found to depend largely on the selected scenario. In this regard, probabilistic optimal power flow (POPF) is a powerful tool for accommodating uncertainties that can yield the statistics of output variables according to the distributions of input variables [18, 19]. Thus, utilizing these output statistics would allow precautions to be formulated in advance.

In addition, for electric power systems employing significant wind power resources, wind power curtailment is unavoidable owing to the fact that variations in wind power generations may be considerable, and, electricity cannot be effectively stored currently [20]. Fortunately, power-to-gas (PtG) technology provides another promising solution to this problem. PtG units convert otherwise curtailed wind power electricity to hydrogen $\left(\mathrm{H}_{2}\right)$ by electrolysis, which may be further converted to synthetic natural gas (SNG) by methanation [21]. The produced $\mathrm{H}_{2}$ or SNG is then blended with natural gas and transported/stored in the natural gas network. In this way, the electric system is further coupled with the gas system by PtG units. Multiple criteria have been proposed for determining the optimal installation location of $\mathrm{PtG}$ units [22]. In addition, a comprehensive technical and economic analysis of $\mathrm{PtG}$ technology has also been conducted [23]. In contrast, few studies have considered the optimization of PtG operations. The present 
study focuses on PtG system operation. Economic viability, and the optimum installation location of PtG units are out of the scope of this paper.

Based on the above discussion, the primary contributions of the present work are as follows:

1) A M-GEOPF model incorporating $\mathrm{PtG}$ units is formulated based on precise nonlinear power and gas flow models, where the dynamical characteristics of the gas network are specifically modeled.

2) To address wind power uncertainty, POPF based on a three-point estimation method (3PEM) is introduced, which, in conjunction with the M-GEOPF model, yields a multi-period gas and electric system probabilistic optimal power flow (M-GEPOPF) model. Additionally, the correlation between gas and electric loads is considered.

3) A probabilistic solution is obtained. By analyzing the probability density of state variables, security precautions can be formulated in advance to mitigate wind power volatility, and more importantly, to avoid gas or electricity system contingencies.

4) The effectiveness of PtG units for accommodating wind power volatility is analyzed. The promising benefits of PtG units are demonstrated in terms of their potential for reducing operational costs and relieving transmission line congestion.

The remainder of this paper is organized as follows. In Sect. 2, the concept of PtG is introduced and a detailed M-GEOPF model is established. The 3PEM-based POPF is presented in Sect. 3, and the correlation between gas and electric loads is considered. Section 4 develops case studies to validate the proposed method, and Sect. 5 draws conclusions from the work.

\section{Modelling of M-GEOPF incorporating power- to-gas units}

In this section, a deterministic M-GEOPF model is established. The two energy systems are treated independently in terms of their physical constraints, and are tightly coupled by gas-fired units and PtG units, enabling a bidirectional energy flow between them.

\subsection{Power-to-gas technology}

Figure 1 presents an illustration of PtG technology and its interaction with gas and electricity networks. As shown in the figure, $\mathrm{PtG}$ units convert surplus renewable energy to $\mathrm{H}_{2}$ by electrolysis. Presently, the highest electrolysis efficiency that can be achieved is $86 \%$ [24]. A portion of $\mathrm{H}_{2}$ is directly injected into the gas network, and the remainder is

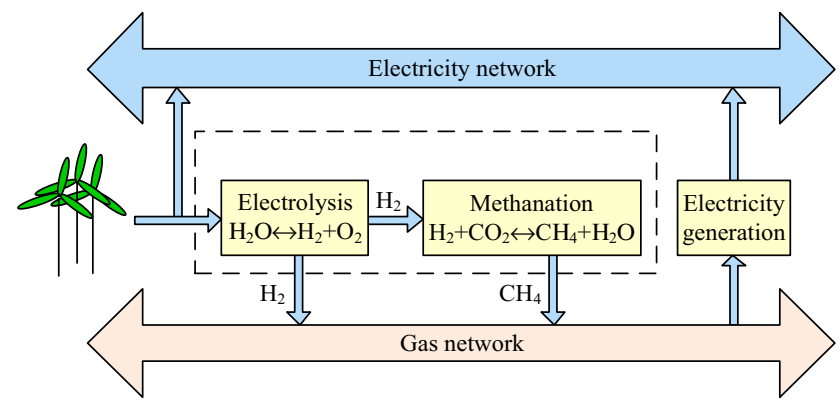

Fig. 1 Power-to-gas technology and its interaction with natural gas and electric power networks

further processed by methanation, which combines $\mathrm{H}_{2}$ with carbon dioxide $\left(\mathrm{CO}_{2}\right)$ to form SNG. The efficiency of this process is about $64 \%$.

Although both $\mathrm{H}_{2}$ and SNG can be blended with natural gas, the storage of $\mathrm{H}_{2}$ involves the following concerns.

1) Safety issues. Reactions between $\mathrm{H}_{2}$ and pipeline materials (such as steel) impose an upper limit on $\mathrm{H}_{2}$ injection.

2) Poorly equipped infrastructure. Existing $\mathrm{H}_{2}$ networks are currently not well-equipped, which renders the transportation of pure $\mathrm{H}_{2}$ problematic.

3) Energy content. The energy content density of $\mathrm{H}_{2}$ is nearly a third that of SNG, indicating that, for an equivalent stored volume of the two gases, the energy content of $\mathrm{H}_{2}$ is far less than SNG.

In view of these considerations, $\mathrm{H}_{2}$ is not discussed in this paper, and is assumed to be processed entirely into SNG. Notice that a PtG unit is a load for electric power system while it is a source for the natural gas system. It is assumed that a PtG unit at bus $i$ of the electric system is connected to node $m$ of the gas system. Then, the relationship between the output gas flow and the input power (relevant definitions provided in the Appendix) is given as follows:

$q_{m, t}^{\mathrm{ptg}}=\frac{P_{i, t}^{\mathrm{ptg}} \eta}{S_{\mathrm{HHV}}}$

where $q_{m, t}^{\mathrm{ptg}}$ is the gas flow converted from the PtG units at node $m$ at time $t ; P_{i, t}^{\mathrm{ptg}}$ is the power consumed by PtG units at bus $i$ at time $t ; \eta$ is the working efficiency of a PtG unit; $S_{\mathrm{HHV}}$ is the higher heating value of SNG $\left(39 \mathrm{MJ} / \mathrm{m}^{3}\right)$.

\subsection{Formulation of M-GEOPF}

The objective of M-GEOPF is to minimize the overall operational costs of the integrated system over a dispatch horizon while satisfying the security constraints of both systems. 


\subsubsection{Objective function}

The objective function consists of power generation costs, power load curtailment costs, natural gas supply costs and gas load curtailment costs. It is noted that the cost of gas-fired units includes the gas consumption cost and the capital and operating generation cost apart from gas consumption. The generation cost is a direct function of power output while the power is converted to gas consumption to account for consumption cost. The objective $S_{\text {obj }}$ is formulated as:

$$
\begin{aligned}
S_{\mathrm{obj}}= & \sum_{t \in \Omega_{\mathrm{T}}}\left(\sum_{g \in \Omega_{\mathrm{gen}}} C_{g}^{\mathrm{gen}} P_{g, t}^{\mathrm{gen}}+\sum_{i \in \Omega_{\mathrm{ele}}} C_{i}^{\mathrm{nsp}} P_{i, t}^{\mathrm{nsp}}+\sum_{i \in \Omega_{\mathrm{ele}}} C_{i}^{\mathrm{wf}} P_{i, t}^{\mathrm{wf}}\right. \\
& \left.+\sum_{w \in \Omega_{\mathrm{we}}} C_{w}^{\mathrm{we}} q_{w, t}^{\mathrm{we}}+\sum_{s \in \Omega_{\mathrm{sto}}} C_{s}^{\mathrm{with}} q_{s, t}^{\mathrm{with}}+\sum_{m \in \Omega_{\mathrm{gas}}} C_{m}^{\mathrm{nsg}} q_{m, t}^{\mathrm{nsg}}\right)
\end{aligned}
$$

where $\Omega_{\mathrm{T}}$ is the set for dispatching periods; $P_{g, t}^{\mathrm{gen}}$ is the active power generation of unit $g$ from generator set $\Omega_{\mathrm{gen}} ; P_{i, t}^{\mathrm{nsp}}$ is the non-served active power at bus $i$ from electricity system bus set $\Omega_{\text {ele }} ; P_{i, t}^{\mathrm{wf}}$ is the wind power that serves electric loads at bus $i$; $q_{w, t}^{\text {we }}$ is gas supply of well $w$ from well set $\Omega_{\mathrm{we}} ; q_{s, t}^{\text {with }}$ is outflow gas rates of storage $s$ from storage set $\Omega_{\text {sto }} ; q_{m, t}^{\text {nsg }}$ is notserved gas at bus $m$ from gas system bus set $\Omega_{\text {gas }} ; C_{g}^{\text {gen }}, C_{i}^{\text {nsp }}$, $C_{i}^{\mathrm{wf}}, C_{w}^{\mathrm{we}}, C_{s}^{\mathrm{with}}$, and $C_{m}^{\mathrm{nsg}}$ are all cost coefficients.

\subsubsection{Electric power system operation constraints}

Day-ahead forecasting of the wind speed is converted to predicted wind power according to the following.

$P_{i, t}^{\mathrm{wf}, \text { fore }}= \begin{cases}0 & v<v_{\mathrm{ci}} \text { or } v>v_{\mathrm{co}} \\ P_{\mathrm{r}}\left(v-v_{\mathrm{ci}}\right) /\left(v_{\mathrm{r}}-v_{\mathrm{ci}}\right) & v_{\mathrm{ci}} \leq v \leq v_{\mathrm{r}} \\ P_{\mathrm{r}} & v_{\mathrm{r}} \leq v \leq v_{\mathrm{co}}\end{cases}$

where $P_{i, t}^{\mathrm{wf} \text {,fore }}$ is total forecasted available wind power at bus $i$; $v$ is wind speed; $v_{\mathrm{ci}}, v_{\mathrm{co}}$, and $v_{\mathrm{r}}$ are cut-in, cut-out and rated wind speed, respectively; $P_{\mathrm{r}}$ is rated wind power.

Multi-period operation constraints for an electric power system include power balance equations, generation capacities, wind power availability, generator ramp rate limits, and voltage and line flow limits, as follows.

$$
\left\{\begin{array}{c}
\sum_{g \in i} P_{g, t}^{\mathrm{gen}}+P_{i, t}^{\mathrm{wf}}+P_{i, t}^{\mathrm{nsp}}-P_{i, t}^{\mathrm{dem}}-P_{i, t}^{\mathrm{ptg}}= \\
V_{i, t} \sum_{j \in i} V_{j, t}\left(G_{i j} \cos \theta_{i j, t}+B_{i j} \sin \theta_{i j, t}\right) \\
\sum_{g \in i} Q_{g, t}^{\mathrm{gen}}+Q_{i, t}^{\mathrm{wf}}-Q_{i, t}^{\mathrm{dem}}= \\
V_{i, t} \sum_{j \in i} V_{j, t}\left(G_{i j} \sin \theta_{i j, t}-B_{i j} \sin \theta_{i j, t}\right)
\end{array}\right.
$$

$$
\begin{aligned}
& P_{g}^{\mathrm{gen}, \mathrm{min}} \leq P_{g, t}^{\mathrm{gen}} \leq P_{g}^{\mathrm{gen}, \mathrm{max}} \\
& Q_{g}^{\mathrm{gen}, \mathrm{min}} \leq Q_{g, t}^{\mathrm{gen}} \leq Q_{g}^{\mathrm{gen}, \mathrm{max}} \\
& 0 \leq P_{i, t}^{\mathrm{wf}}+P_{i, t}^{\mathrm{ptg}} \leq P_{i, t}^{\mathrm{wf}, \text { fore }} \\
& R^{\mathrm{dn}} \leq P_{g, t}^{\mathrm{gen}}-P_{g, t-1}^{\mathrm{gen}} \leq R^{\mathrm{up}} \\
& V_{i}^{\mathrm{min}} \leq V_{i, t} \leq V_{i}^{\mathrm{max}} \\
& \left|-V_{i, t}^{2} G_{i j}+V_{i, t} V_{j, t}\left(G_{i j} \cos \theta_{i j, t}+B_{i j} \sin \theta_{i j, t}\right)\right| \leq P_{i j}^{\max }
\end{aligned}
$$

where $P_{i, t}^{\mathrm{dem}}$ is the active electric demand at bus $i ; V_{i, t}$ is the voltage magnitude of bus $i ; \theta_{i j, t}$ is the difference of voltage angle between bus $i$ and bus $j ; G_{i j}, B_{i j}$ are the conductance and susceptance between bus $i$ and bus $j$, respectively; $Q_{g, t}^{\text {gen }}$ is the reactive power generation of the unit $g ; Q_{i, t}^{\mathrm{wf}}$ is the reactive wind power generation at bus $i ; Q_{i, t}^{\mathrm{dem}}$ is the reactive electric demand at bus $i ; P_{g}^{\text {gen,min }}$ and $P_{g}^{\text {gen,max }}$ are minimum and maximum active power generation, respectively; $Q_{g}^{\text {gen,min }}$ and $Q_{g}^{\text {gen,max }}$ are minimum and maximum reactive power generation, respectively; $R^{\mathrm{dn}}$ and $R^{\text {up }}$ are upward and downward ramp rate for conventional generators, respectively; $V_{i}^{\min }$ and $V_{i}^{\max }$ are minimum and maximum voltage magnitude, respectively; $P_{i j}^{\max }$ is the maximum power line flow.

It is noted that $P_{i, t}^{\mathrm{wf}}$ denotes the wind power that serves electric loads, $P_{i, t}^{\mathrm{ptg}}$ denotes the surplus wind power to be converted to natural gas, and $P_{i, t}^{\mathrm{wf}, \text { fore }}$ denotes the total forecasted available wind power. Thus, (7) indicates that the wind power consumed by either PtG units or electric loads should not exceed its forecasted availability.

\subsubsection{Natural gas system operation constraints}

The natural gas system consists of gas wells, storage facilities, pipelines, compressors, and valves. Natural gas, beginning at a distant gas well or a remote storage facility, flows into the distribution network, through which it is delivered to customers. The physical constraints on gas supply from wells are given by (11), and from storage facilities by (12) and (13).

$q_{w}^{\mathrm{we}, \min } \leq q_{w, t}^{\mathrm{we}} \leq q_{w}^{\mathrm{we}, \max }$

$l_{s}^{\min } \leq l_{s, t}=l_{s, t-1}+q_{s, t}^{\text {inj }}-q_{s, t}^{\text {with }} \leq l_{s}^{\max }$

$q_{s, t}^{\mathrm{inj}} \leq q_{s}^{\mathrm{inj}, \max }, \quad q_{s, t}^{\text {with }} \leq q_{s}^{\text {with,max }}$

where $q_{w}^{\text {we,min }}$ and $q_{w}^{\text {we,max }}$ are minimum and maximum gas flow from gas well $w$, respectively; $l_{s, t}$ is the volume of storage $s ; q_{s, t}^{\text {inj }}$ is the gas inflow to storage $s$ (or withdrawal rate if negative); $l_{s}^{\min }$ and $l_{s}^{\max }$ are minimum and maximum 
storage capacities, respectively; $q_{s}^{\text {inj,max }}$ and $q_{s}^{\text {with,max }}$ are the maximum injection and withdrawal rates of storage $s$.

Pipelines represent major components in natural gas infrastructure. The flow rate in pipeline $m-n$ depends on the pressure difference between inlet node $m$ and outlet node $n$ :

$\widetilde{q}_{m n, t}\left|\widetilde{q}_{m n, t}\right|=\left(\frac{\pi}{4}\right)^{2} \frac{D_{m n}^{5}}{\Delta x_{m n} F_{m n} R T Z \rho_{0}^{2}}\left(p_{m, t}^{2}-p_{n, t}^{2}\right)$

where $D_{m n}$ is the pipeline diameter of pipeline $m-n ; \Delta x_{m n}$ is the length of pipeline $m-n ; F_{m n}$ is the friction coefficient of pipeline $m-n ; R$ is the specific gas constant $(56 \mathrm{~J} /(\mathrm{kg} \cdot \mathrm{K})) ; T$ is the gas temperature; $Z$ is the gas compression factor; $\rho_{0}$ is natural gas density under standard conditions $(0.713 \mathrm{~kg} /$ $\left.\mathrm{m}^{3}\right) ; p_{m, t}$ is the pressure at node $m ; \widetilde{q}_{m n, t}=\left(q_{m n, t}^{\text {out }}+q_{m n, t}^{\text {in }}\right) / 2$ is the average gas flow rate in pipeline $m-n$. The absolute value sign denotes that gas flow can be bi-directional, which depends on the pressure difference. However, due to the compressibility of natural gas, the quantity of inlet and outlet flow in a pipe may differ, making it possible to store gas in a pipeline during off-peak load periods and withdraw when necessary. This is called "line pack" and it fosters more flexible operation of the integrated system. Line pack is defined as follows [13]:

$\widetilde{l p}_{m n, t}=\widetilde{l p}_{m n, t-1}+q_{m n, t}^{\mathrm{in}}-q_{m n, t}^{\mathrm{out}}=\frac{\pi}{4} \frac{\Delta x_{m n} D_{m n}^{2}}{R T_{\mathrm{em}} Z \rho_{0}} \frac{p_{m, t}+p_{n, t}}{2}$

where $\widetilde{l p}_{m n, t}$ is average gas mass (line pack) of pipeline $m$ $n ; q_{m n, t}^{i n}$ and $q_{m n, t}^{\text {out }}$ are inflow and outflow gas rates of pipeline $m-n$, respectively; $T_{\mathrm{em}}$ is average temperature (288 K).

Equations (11)-(15) account for the gas dynamic characteristics by taking gas velocity and line pack into consideration. Gas velocity is eliminated in the process of deriving (14) and (15) [13]. Compressors are deployed to compensate for gas pressure loss suffered during transport due to frictional resistance. Correspondingly, a large amount of power is required to increase the pressure level. This power is usually provided by a gas turbine, conveniently taking the advantage of the transported natural gas, although steam or electricity could also be used [25]. The horsepower consumed by the compressor is determined by the transported gas flow and the compression ratio as given in (16) [7]. For a gas-driven compressor, additional gas would be extracted from gas network to supply the horsepower as given in (17).

$H_{c, t}=B_{c} q_{c, t}\left[\left(p_{n, t} / p_{m, t}\right)^{Z_{c}}-1\right]$

$\tau_{c, t}=\alpha+\beta H_{c, t}+\gamma H_{c, t}^{2}$ where $H_{c, t}$ is the horsepower consumed by a gas-turbine to drive compressor $c ; B_{c}$ is the compressor constant; $q_{c, t}$ is gas flow of compressor $c ; Z_{c}$ is the compressor factor $(0.95) ; \tau_{c, t}$ is gas consumption of a gas-turbine to drive a compressor; $\alpha, \beta$ and $\gamma$ are all energy conversion constants.

Gas nodal pressures should be operated within secure ranges. For nodes connected with a compressor, we use (18), while (19) corresponds to the other gas nodes.

$p_{n, t} \leq \Gamma_{c} p_{m, t}$

$p_{m}^{\min } \leq p_{m, t} \leq p_{m}^{\max }$

where $\Gamma_{c}$ is maximum compression ratio of compressor $c$; $p_{m}^{\min }$ and $p_{m}^{\max }$ are the minimum and maximum node pressure, respectively.

Finally, the following nodal gas flow balance equation should be satisfied for each node.

$$
\begin{array}{r}
\sum_{n \in m}\left(q_{m n, t}^{\mathrm{out}}-q_{m n, t}^{\mathrm{in}}\right)+\sum_{c \in m}\left(q_{c, t}+\tau_{c, t}\right)+\sum_{w \in m} q_{w, t}^{\mathrm{we}}+q_{m, t}^{\mathrm{ptg}} \\
+\sum_{s \in m}\left(q_{s, t}^{\mathrm{with}}-q_{s, t}^{\mathrm{inj}}\right)=q_{m, t}^{\mathrm{dem}}-q_{m, t}^{\mathrm{nsg}}+\sum_{g \in m} P_{g, t}^{\mathrm{gen}} \cdot \phi_{g}
\end{array}
$$

where $\phi_{g}$ is the energy conversion coefficient for gas turbines.

In (20), when $m$ is the inlet node of compressor $c, q_{c, t}$ and $\tau_{c, t}$ are positive, and when $m$ is the outlet node of compressor $c, q_{c, t}$ is negative and $\tau_{c, t}$ is zero. The last term on the right-hand side of (20) denotes the relationship between gas consumption and power generation of gasfired units.

The proposed M-GEOPF model is a deterministic optimization problem, in which the control variables include active and reactive power generation, curtailed wind power, gas supply, and load curtailments. This combined optimization model can be solved via commercial optimization tools to achieve optimal results.

\section{M-GEPOPF based on the three-point estimation method}

The considered integrated energy system is faced with remarkable uncertainties originating from electricity/gas load forecasting errors and intermittent wind power generations. As such, we propose POPF to investigate the impacts of these uncertainties on the operation of integrated electric and gas systems. The previously proposed three-point estimation method [26] is deployed in our work. 


\subsection{Three-point estimation method (3PEM)}

The target of point estimation is to calculate the moments of output variable vector $\boldsymbol{W}$ with respect to $l$ input variables. In other words, the state of the system is stochastic as a nonlinear function of the input vector $\boldsymbol{X}$, written as:

$\boldsymbol{W}=\boldsymbol{G}(\boldsymbol{X})=\boldsymbol{G}\left(x_{1}, x_{2}, \ldots, x_{l}\right)$

The fundamental idea of point estimation lies in matching the first several moments of each stochastic variable $x_{b}$ with $k$ points. In this paper, the 3PEM, i.e., $k=3$, is adopted owing to its easy implementation, computational efficiency and high accuracy. According to the 3PEM, three concentrations, each of which consists of a location $\xi_{x_{b, k}}$ and a weighting factor $\omega_{x_{b, k}}(k=1,2,3)$, are computed for each $x_{b}$ as follows.

$x_{b, k}=\mu_{x_{b}}+\xi_{b, k} \sigma_{x_{b}}$

$\left\{\begin{array}{c}\omega_{x_{b, k}}=\frac{(-1)^{3-k}}{\xi_{x_{b, k}}\left(\xi_{x_{b, 1}}-\xi_{x_{b, 2}}\right)}(k=1,2), \omega_{x_{b, 3}}=\frac{1}{l}-\frac{1}{v_{x_{i}}-\lambda_{x_{i}}^{2}} \\ \xi_{x_{b, k}}=\frac{\lambda_{x_{b}}}{2}+(-1)^{3-k} \sqrt{v_{x_{b}}-\frac{3}{4} \lambda_{x_{b}}^{2}}(k=1,2), \xi_{x_{b, 3}}=0\end{array}\right.$

where $\mu_{x_{b}}$ and $\sigma_{x_{b}}$ are the mean value and standard deviation of variable $x_{b}$, respectively; $v_{x_{i}}$ and $\lambda_{x_{i}}$ are the skewness and kurtosis of variable $x_{b}$, respectively.

Finally, by aggregating all the weighted deterministic results, the $r$-th estimated raw moment of $\boldsymbol{W}$ can be calculated as:

$E\left(\boldsymbol{W}^{r}\right) \cong \sum_{b=1}^{l} \sum_{k=1}^{3} \omega_{x_{b, k}} \times\left[\boldsymbol{G}\left(\mu_{x_{1}}, \mu_{x_{2}}, \ldots, x_{b, k}, \mu_{x_{l}}\right)\right]^{r}$

where $\mathrm{E}(\cdot)$ denotes the expectation operator.

In our study, electric load uncertainty, gas load uncertainty, and wind speed uncertainty are considered. However, considering the load uncertainty at each bus or node in each period would represent a tremendous computational burden. An excessive number of simulation runs are required because of numerous electrical/gas nodes and optimization horizon. Yet, the fact is, the load variation of a given bus or node has negligible impact on the overall result. Hence, we consider the randomness of the total load at each time period as a whole. The input stochastic variable is denoted as $\boldsymbol{X}=\left[\boldsymbol{P}^{\mathrm{dem}}, \boldsymbol{P}^{\mathrm{wf} \text {,fore }}, \boldsymbol{q}^{\mathrm{dem}}\right]^{\mathrm{T}}$ and the output is denoted as $\boldsymbol{W}=\left[\boldsymbol{V}, \boldsymbol{\theta}, \boldsymbol{P}^{\text {gen }}, \boldsymbol{Q}^{\text {gen }}, \boldsymbol{p}, \boldsymbol{q}^{\text {we }}, \boldsymbol{q}^{\text {inj }}, \boldsymbol{q}^{\text {with }}, \boldsymbol{q}^{\text {in }}, \boldsymbol{q}^{\text {out }}\right]^{\mathrm{T}}$.

\subsection{Correlation between gas and electric loads}

In reality, a correlation exists between gas and electric loads, which can be addressed by the 3PEM. Assuming that the cumulative distribution function (CDF) of $x_{b}$ is $F_{b}\left(x_{b}\right)$,
Start

$\downarrow$

\begin{tabular}{|} 
Input system parameters, including the statistics of gas load, \\
electric load, and wind power
\end{tabular}



Fig. 2 Flow chart of M-GEPOPF calculation

the standard normal vector $\boldsymbol{Y}=\left[y_{1}, y_{2}, \ldots, y_{l}\right]$ can be obtained from $\boldsymbol{X}$ according to:

$y_{b}=\Phi^{-1}\left(F_{b}\left(x_{b}\right)\right)$

where $\Phi^{-1}(\cdot)$ denotes the inverse CDF of the standard normal variable. Meanwhile, $\boldsymbol{Y}$ can be converted to an independent standard normal vector $\boldsymbol{Z}$. Let $\boldsymbol{C}$ denote the linear correlation coefficient of $\boldsymbol{X}$. The coefficient matrix $\boldsymbol{C}$ can be decomposed into a lower triangular matrix $\boldsymbol{B}$ by Cholesky decomposition. Thus, $\boldsymbol{Z}$ can be computed as $\boldsymbol{Z}=\boldsymbol{B}^{-1} \boldsymbol{Y}$.

Generally, when correlation exists among input variables, it is advisable to sample in the independent standard normal space, and then transform the sample points into the original state space. The flow chart of the proposed M-GEPOPF is presented in Fig. 2.

\section{Case study}

In this section, we discuss the application of M-GEPOPF to a test system. The impact of the correlation between gas and electric loads on the probabilistic results is investigated. Additionally, the role of $\mathrm{PtG}$ units is demonstrated. 


\subsection{System description}

A schematic illustrating the integrated IEEE 24-bus electric power system and Belgian high-calorific 20-node natural gas system employed in the case study is given in Fig. 3. All detailed data on the tested system is available in [12]. In addition, a wind farm with an $800 \mathrm{MW}$ rated power is connected to the electricity system at bus 8 . Wind speed data were obtained from the National Renewable Energy Laboratory (NREL) for the site \#1 wind farm [27]. The gas system consists of 20 nodes, 21 pipelines, 2 compressors, 2 gas wells, and 4 gas storage facilities. A PtG unit is deployed near the wind farm connected at bus 8 , and is integrated with the gas system at node 16 (i.e., the Blaregnies node), which generally faces heavy gas demand.

Gas and electric loads at each period are assumed to conform to a normal distribution, taking the forecasted value as its mean and $2 \%$ of the forecasted value as its standard deviation. In addition, the wind speed at each time interval is usually assumed to comply with a normal distribution [26], taking the forecasted value as its mean and $5 \%$ of the forecasted value as its standard deviation.

\subsection{Accuracy clarification}

The proposed model is a large-scale nonlinear programming (NLP) problem. Advanced solvers have been developed so as to achieve a sufficiently optimal solution. The present NLP problem was conducted on the GAMS platform using the NLP solver IPOPT [28]. The Monte Carlo method with a sample of 5000 is adopted as a reference to investigate the performance of the 3PEM, in which all input variables are randomly generated from a predetermined distribution. The M-GEPOPF computation based on the 3PEM required approximately $430 \mathrm{~s}$ for the case study system, which is quite acceptable for a system of this size.

For each time interval, the statistics of each bus voltage and node pressure are calculated and averaged. A comparison between the 3PEM and Monte Carlo results is depicted in Fig. 4. Here, Fig. 4a presents comparisons of the mean value and the standard deviation of the electric system bus voltage. The percentage errors are found to be rather small, where the mean value error is less than $0.015 \%$ and the standard deviation error is $4 \%$. Figure $4 \mathrm{~b}$ presents comparisons of natural gas system node pressure, where the errors are again acceptable. In this way, the accuracy of the 3PEM has been verified.

\subsection{Role of PtG units}

To evaluate the role of PtG units, we compare the test system with and without the single PtG unit. Figure $4 a, b$ depicts the wind power utilization by the electricity transmission system or by distribution to the $\mathrm{PtG}$ unit. Because electricity transmission lines have limited capacity, congested transmission lines during peak load periods makes it difficult to transfer all generated wind power from bus 8 . As a result, it can be observed that wind power is mainly curtailed from 3:00 to 14:00. Making use of the PtG unit allows the available wind power to be fully exploited to support the natural gas network. Moreover, we compare

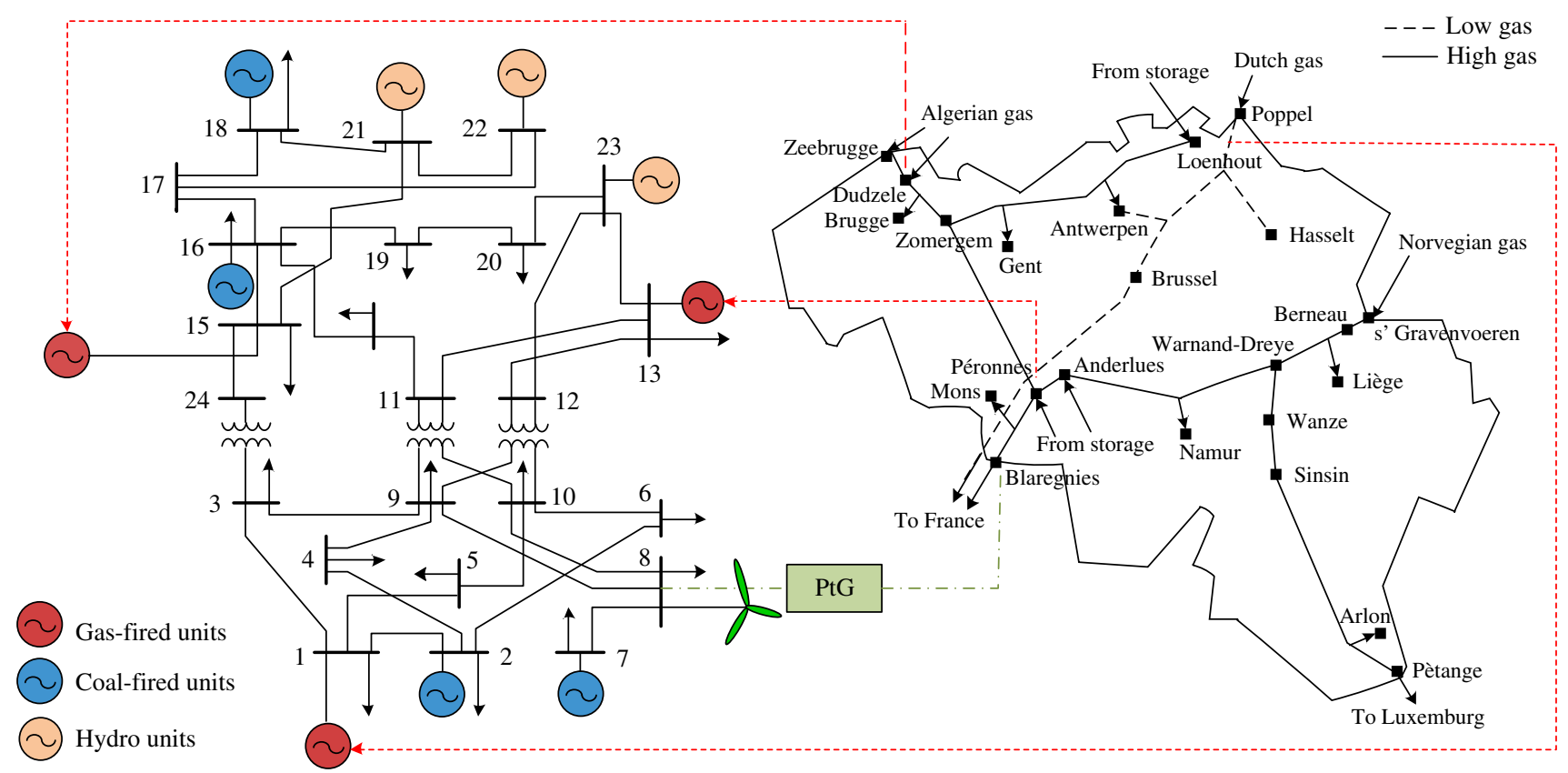

Fig. 3 Schematic of integrated gas and electric system 


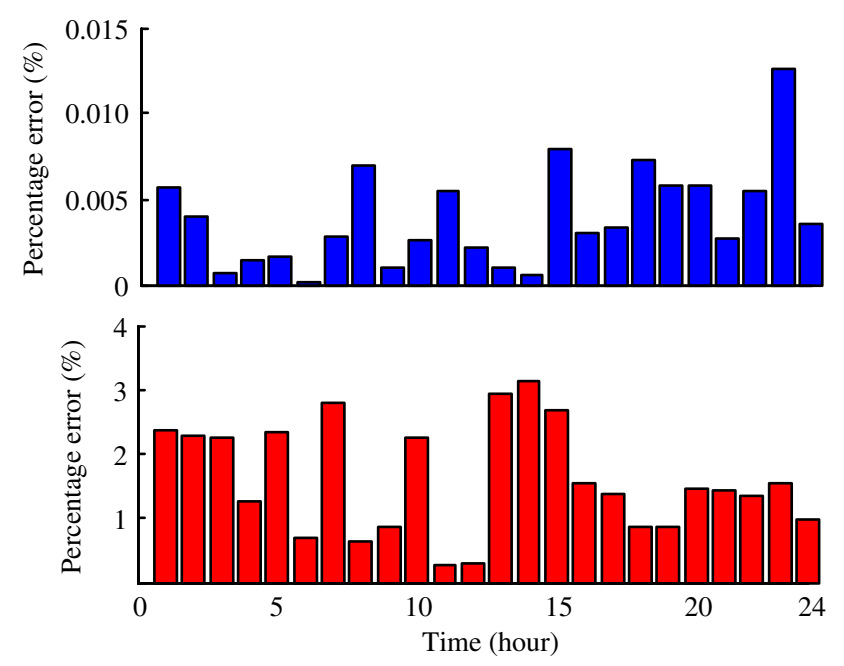

(a) Bus voltage percentage error

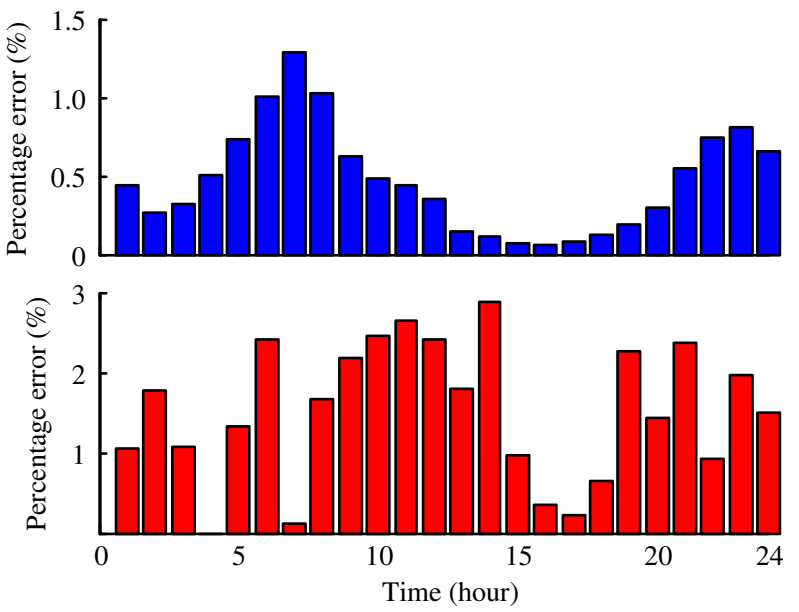

(b) Node pressure percentage error

Fig. 4 Comparison between 3PEM and Monte Carlo simulation results

in Fig. 5c the extent to which wind power is integrated with the electricity system for generation with and without the PtG unit. Including a PtG unit allows greater wind power to be absorbed by the electricity network. This is because transmission line congestion is effectively relieved, enabling the electricity network to accommodate much more wind power generation.

The produced SNG is consumed locally or transported, and, due to its zero cost assumption, reduced total operation costs can be expected. Not surprisingly, the total daily operational costs for the system with and without a PtG unit are \$ 6700 kilo and \$ 6788 kilo, respectively. Moreover, additional economic benefits derive from two sources. First, natural gas consumption from gas wells or gas storage facilities is reduced by utilizing gas produced from $\mathrm{PtG}$ units. Second, generation costs from conventional power plants are reduced owing to the increased adoption of wind power generation.

\subsection{Probabilistic analysis of correlation}

The optimization results of the integrated energy system are shown in Fig. 6. The expected power outputs of hydroelectric units, coal-fired units, gas-fired units, and wind farms are depicted in Fig. 6a. Hydroelectric units are fully utilized over the entire 24 hours period because of their economic efficiency. In contrast, coal-fired units are always operated at their lowest generation level. The flexibility of gas-fired units facilitates their role of output adjustment. While the cost of wind power is nearly zero, it is not fully utilized because of electric line congestion near the wind farm. Moreover, there are two electricity load peaks, and the output of gas-fired power plants at the first load peak is apparently less than that at the second load peak. This is because the first electric load peak coincides with a gas demand peak, and this constraints the availability of gas supply gas-fired units during this period. This result clearly exemplifies the necessity for optimizing gas and electric systems from an integrated point of view.

The optimization results for the gas system are shown in Figs. $6 \mathrm{~b}, \mathrm{c}$, which present the expectations of gas production and line pack respectively. In our study, gas wells are the main gas source, and gas derived from storage facilities

Table 1 Statistics of total operation cost with different gas-electricity correlation coefficients

\begin{tabular}{lll}
\hline Gas-electricity correlation coefficient & $\begin{array}{l}\text { Mean of total operational cost } \\
(\text { kilo } \$)\end{array}$ & $\begin{array}{l}\text { Standard deviation of total operational cost } \\
\text { (kilo· } \$)\end{array}$ \\
\hline 0.8 & 6700 & 51.9 \\
0.5 & 6700 & 51.3 \\
0.3 & 6700 & 50.8 \\
0 & 6700 & 50.2 \\
-0.3 & 6700 & 49.5 \\
-0.5 & 6700 & 49.1 \\
-0.8 & 6700 & 48.4 \\
\hline
\end{tabular}




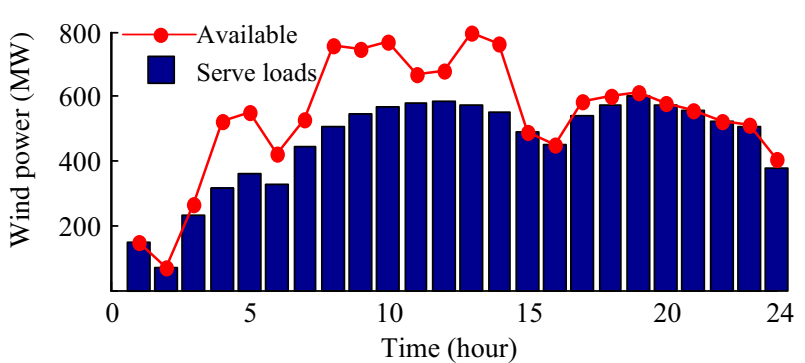

(a) Wind power utilization without PtG

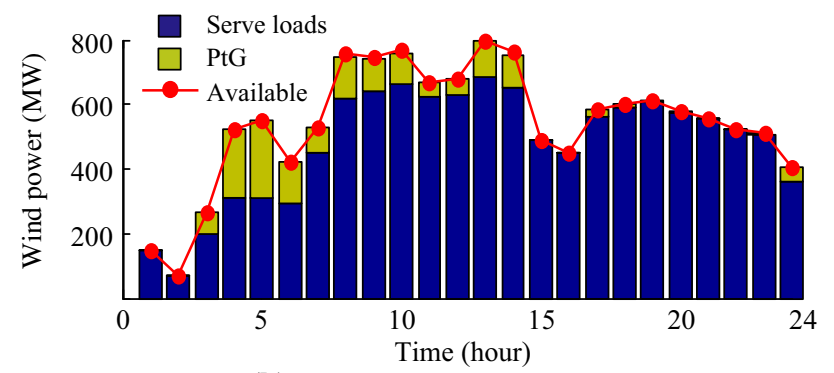

(b) Wind power utilization with PtG

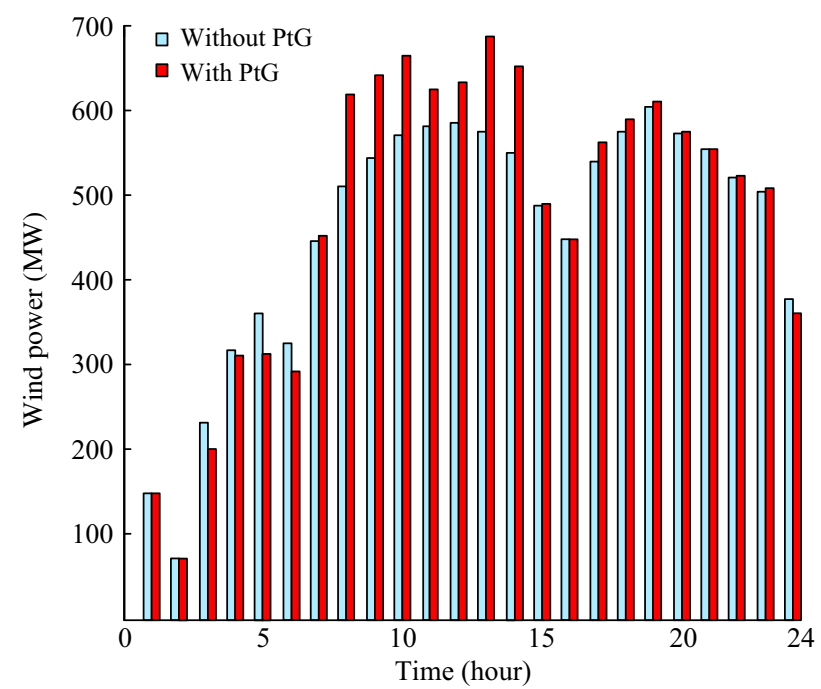

(c) Wind power integrated into the electric network for serving electric loads

Fig. 5 Analysis of role of PtG

is employed over all periods owing to its economic efficiency. In addition, Fig. 6c indicates that line pack also serves as a means of gas storage; indeed, the range of line pack exceeds the combined range of well production and gas storage over the modelled period. Two gas well supply peaks are shown in Fig. 6b, where the first peak corresponds to a gas demand peak and the second peak corresponds to a power supply peak. Moreover, gas is stored by means of line pack from 1:00 to 6:00, when the gas load is relatively low, and the line pack later releases gas in response to increasing gas demand. Thus, the line pack also enables flexible operation of the integrated system.

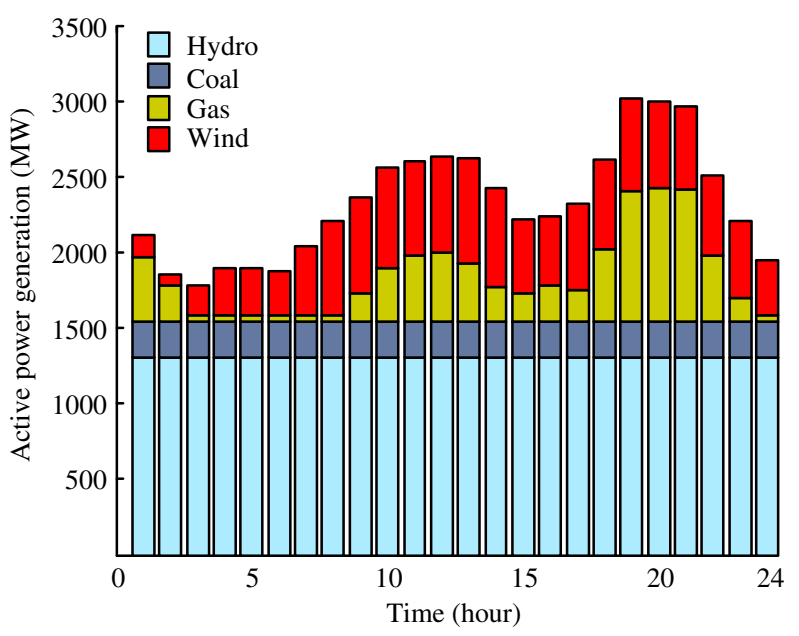

(a) Expectation of power generation

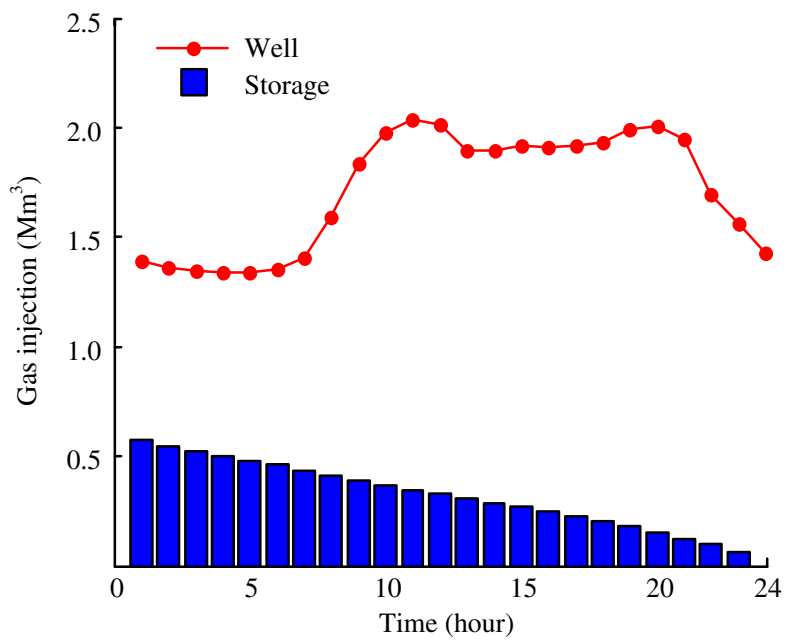

(b) Expectation of well production and gas storage

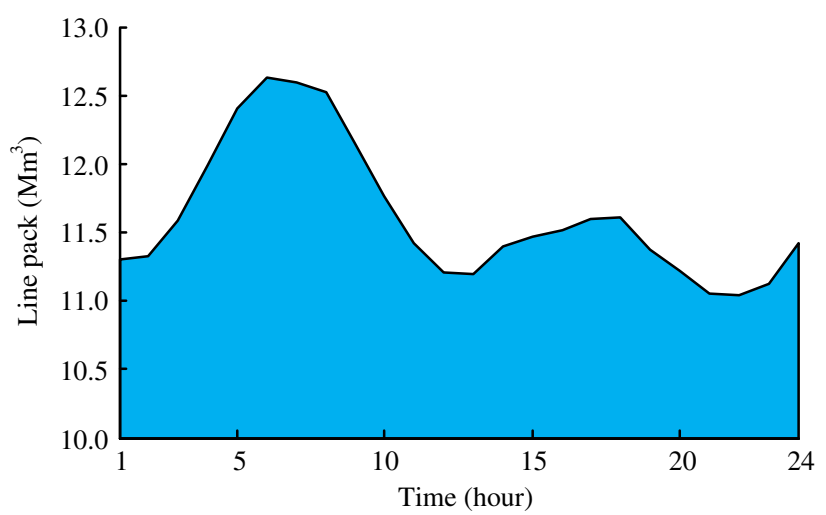

(c) Expectation of line pack

Fig. 6 Optimization results of integrated energy system

M-GEPOPF can provide system operators with an exact measure of the statistical properties of state variables (e.g., bus voltages and nodal pressures) that must be carefully monitored. Figure $7 \mathrm{a}$ presents the probability density function (PDF) of voltage at bus 6, near to where the wind farm and PtG plant are located, at 12:00. There is an 


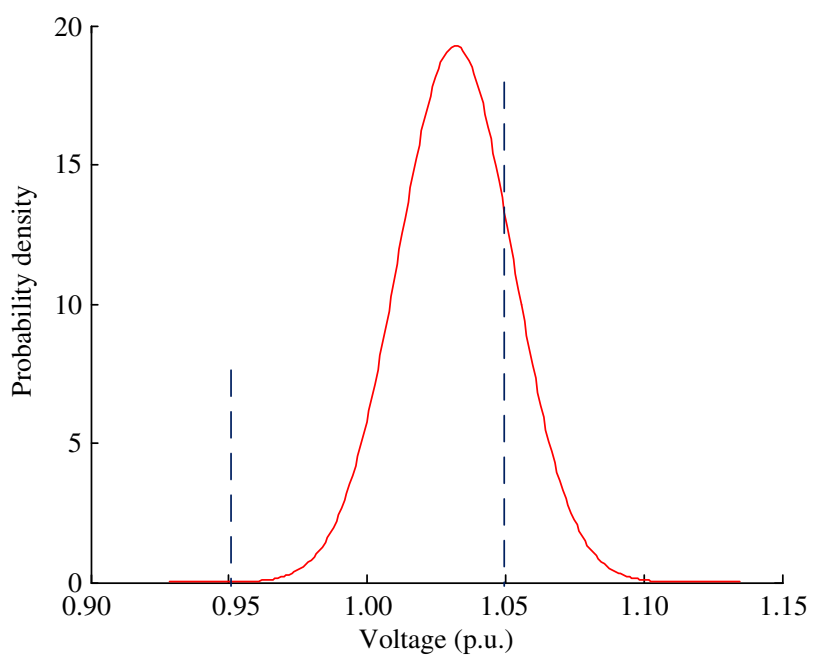

(a) PDF of bus 6 voltage at 12:00



(b) PDF of node 16 pressure at 12:00

Fig. 7 PDF of system state variables at 12:00

electric peak at 12:00, and fluctuations of bus 6 are relatively high at this moment (in this case lower and upper bounds of bus 6 voltage are 0.95 and 1.05 p.u., respectively). The probability that the voltage of bus 6 exceeds security region is $19.1 \%$ which is significant. Similarly, Fig. $7 \mathrm{~b}$ denotes the PDF of node 16 pressure, where the $\mathrm{PtG}$ is integrated at this node (lower and upper bounds of node 16 pressure are 50 bar and 66.2 bar, respectively). According to this figure, the probability that this nodal pressure exceeds its permissive limit is slight $(0.6 \%)$. Other statistics can be achieved in the same way. Operational risk should be addressed by using safety precautions. It is noted that the electric power system endures a more volatile situation owing to the more significant uncertainty of wind power. In addition, the correlation between electric power and natural gas loads should also be adequately analysed in the M-GEPOPF calculation. Table 1 presents the mean and standard deviation of the total operational costs with different gas-electricity correlation coefficients.

The first column in Table 1 represents the elements of the correlation matrix $\boldsymbol{C}$. It is observed from Table 1 that the correlation between gas and electric loads has no discernible impact on the mean value of the total operational costs, while correlation has a more significant impact on their standard deviation. Here, a positive correlation between gas and electric loads results in a larger standard deviation in the total operational costs with increasing correlation, however, an increasingly negative correlation results in a decreasing standard deviation. It is easy to understand why this should be so; positive correlation intensifies the uncertainty in the integrated energy system, whereas an increase in one load correlated with a decrease in the other, i.e., negative correlation, mitigates the volatility via compensation between the gas and electric networks. If correlation is omitted in conventional POPF, the obtained results may lose their optimality. Consequently, it is imperative to include randomness and correlation in M-GEPOPF calculations in order to obtain a proper understanding of the combined system costs and its security regime.

\section{Conclusion}

This paper proposed an M-GEPOPF model incorporating PtG units. The 3PEM based POPF calculation was adopted to address wind power uncertainty. Correlation between gas and electric loads was also investigated, and was shown to exert a significant influence on the probabilistic results. M-GEPOPF calculations demonstrated that the independent optimization of gas and electric network may lead to an overly-optimistic, or even unreliable solution; thus, compromising system security. Based on the probability density of state variables, security precautions can be formulated in advance to mitigate fluctuations in wind power.

In addition, the role of PtG units was discussed. Analysis demonstrated that $\mathrm{PtG}$ units contribute to reducing wind power curtailment by relieving electricity transmission line congestion, thus reducing system operational costs, and increasing the ability of the power system to integrated wind power. The proposed M-GEPOPF model provides a useful support for system operations and decisions.

It must be noted that gas derived from PtG units can be stored to shave the electric peaks in response to variable electricity prices, which is an interesting topic not in the scope of the present study. This will also be investigated in our future research. 
Acknowledgements This work is supported by the National Natural Science Foundation of China (No. 51277052, No. 51407125).

Open Access This article is distributed under the terms of the Creative Commons Attribution 4.0 International License (http:// creativecommons.org/licenses/by/4.0/), which permits unrestricted use, distribution, and reproduction in any medium, provided you give appropriate credit to the original author(s) and the source, provide a link to the Creative Commons license, and indicate if changes were made.

\section{References}

[1] Fact sheet: clean power plan key changes and improvement. United States Environmental Protection Agency. http://www2. epa.gov/cleanpowerplan/fact-sheet-clean-power-plan-keychanges-and-improvements. Accessed 20 Sept 2017

[2] International energy outlook. http://www.eia.gov/outlooks/ieo/ pdf/0484(2016).pdf. Accessed 20 Sept 2017

[3] Soltan S, Mazauric D, Zussman G (2014) Cascading failures in power grids: analysis and algorithms. In: International conference on future energy systems. ACM, pp 195-206

[4] Xue Y (2015) Energy internet or comprehensive energy network? J Mod Power Syst Clean Energy 3(3):297-301. doi:10. 1007/s40565-015-0111-5

[5] Jing Q, Dong ZY, Zhao JH (2015) Low carbon oriented expansion planning of integrated gas and power systems. IEEE Trans Power Syst 30(2):1035-1046

[6] Urbina M, Li Z (2008) Modeling and analyzing the impact of interdependency between natural gas and electricity infrastructures. In: Proceedings of IEEE/PES general meeting, Pittsburgh, PA, USA, 20-24 Jul. 2008, 6 pp

[7] An S, Li Q, Gedra TW (2003) Natural gas and electricity optimal power flow. In: Proceedings of IEEE/PES transmission and distribution conference and exposition, Dallas, TX, USA, 7-12 Sep. 2003, 6 pp

[8] Unsihuay C, Lima JWM, De Souz ACZ (2007) Modeling the integrated natural gas and electricity optimal power flow. In: Proceedings of IEEE/PES general meeting, Jun. 2007, 5 pp

[9] Liu C, Shahidehpour M, Wang J (2010) Application of augmented lagrangian relaxation to coordinate scheduling of interdependent hydrothermal power and natural gas systems. IET Gener Transm Distrib 4(12):1314-1325

[10] Chaudry M, Jenkins N, Strbac G (2008) Multi-time period combined gas and electricity network optimization. Electr Power Syst Res 78(7):1265-1279

[11] Qadrdan M, Chaudry M, Wu J et al (2010) Impact of a large penetration of wind generation on the GB gas network. Energy Policy 38(10):5684-5695

[12] Liu C, Shahidehpour M, Wang J (2011) Coordinated scheduling of electricity and natural gas infrastructures with a transient model for natural gas flow. Chaos Interdiscip J Nonlinear Sci 21(2):025102-025102

[13] Correa-Posada CM, Sanchez-Martin P (2015) Integrated power and natural gas model for energy adequacy in short-term operation. IEEE Trans Power Syst 30(6):3347-3355

[14] Guandalini G, Campanari S, Romano MC (2015) Power-to-gas plants and gas turbines for improved wind energy dispatchability: energy and economic assessment. Appl Energy 147:117-130

[15] Martinez-Mares A, Fuerte-Esquivel CR (2013) Interdependency analysis of integrated energy systems considering wind power uncertainty. IEEE Trans Power Syst 28(4):3964-3976
[16] Parisio A, Del Vecchio C, Vaccaro A (2012) A robust optimization approach to energy hub management. Int $\mathrm{J}$ Electr Power Energy Syst 42(1):98-104

[17] Qadrdan M, Wu J, Jenkins N et al (2014) Operating strategies for a GB integrated gas and electricity network considering the uncertainty in wind power forecasts. IEEE Trans Sustain Energy 5(1):128-138

[18] Wei ZN, Chen S, Sun GQ et al (2016) Probabilistic available transfer capability calculation considering static security constraints and uncertainties of electricity-gas integrated energy systems. Appl Energy 167:305-316

[19] Qadrdan M, Abeysekera M, Chaudry M et al (2015) Role of power-to-gas in an integrated gas and electricity system in Great Britain. Int J Hydrogen Energy 40(17):5763-5775

[20] Hitaj C (2015) Location matters: The impact of renewable power on transmission congestion and emissions. Energy Policy $86: 1-16$

[21] Gahleitner G (2013) Hydrogen from renewable electricity: An international review of power-to-gas plants for stationary applications. Int J Hydrogen Energy 38(5):2039-2061

[22] Moskalenko N, Lombardi P, Komarnicki P (2014) Multi-criteria optimization for determining installation locations for the power-to-gas technologies. In: PES general meeting / conference \& exposition, 2014 IEEE, Washington, DC, USA, Jul. 2014, 5 pp

[23] Belderbos A, Delarue E, D'haeseleer W (2015) Possible role of power-to-gas in future energy systems. In: European Energy Market (EEM), 2015 12th international conference on the IEEE, Lisbon, Portugal, May 2015, 5 pp

[24] RWE Power-to-Gas Energy Storage System Lauched. ITM Power. http://www.itm-power.com/project/gridgas

[25] Acha S (2013) Modelling distributed energy resources in energy service network. IET Press, London

[26] Saunders CS (2014) Point estimate method addressing correlated wind power for probabilistic optimal power flow. IEEE Trans Power Syst 29(3):1045-1054

[27] Jager D, Andreas A (1996) NREL Report No. DA-5500-56489. http://dx.doi.org/10.5439/1052222. Accessed 20 Sept 2017

[28] Wächter A, Biegler LT (2006) On the implementation of an interiorpoint filter line-search algorithm for large-scale nonlinear programming. Math Program 106(1):25-57

Guoqiang SUN received the B.S., M.S., and Ph.D. degrees in electrical engineering from Hohai University, Nanjing, China, in 2001, 2005, and 2010, respectively. He was a visiting scholar at North Carolina State University, Raleigh, NC from 2015 to 2016. He is now an Associate Professor with the College of Energy and Electrical Engineering, Hohai University, Nanjing, China. His research interests include power system analysis and economic dispatch and optimal control of integrated energy systems.

Shuang CHEN received her Bachelor degree from the College of Energy and Electrical Engineering, Hohai University, Nanjing, China, in 2014, and now she is pursuing the M.S. degree. Her research interests include the integration of renewable energies into power system and optimal power flow of integrated energy systems.

Zhinong WEI received the B.S. degree from Hefei University of Technology, Hefei, China, in 1984, the M.S. degree from Southeast University, Nanjing, China, in 1987, and the Ph.D. degree from Hohai University, Nanjing, China, in 2004. He is now a Professor of electrical engineering with the College of Energy and Electrical Engineering, Hohai University, Nanjing, China. His research interests include power system state estimation, integrated energy systems, 
smart distribution systems, optimization and planning, load forecasting, and integration of distributed generation into electric power systems.

Sheng CHEN received his Bachelor degree from the College of Energy and Electrical Engineering, Hohai University, Nanjing, China, in 2014, where he is currently pursuing the Ph.D. degree. His research interests include economic dispatch and security analysis of integrated energy systems. 